\title{
My constant companions
}

fter more than 30 years working at The Aerospace A Corporation in Los Angeles, I retired in 2017. Shortly thereafter, I moved to Arkansas. Although I am surrounded by family and friends, with whom I interact regularly, my constant companions for the last few years have been my iPhone and iPad. One or both of them are with me almost everywhere I go.

My life would be much less comfortable without these devices. The iPhone is my main telephone link, which I use extensively for talking and texting. FaceTime and social media, that includes Facebook and In-stagram, allow me to keep in touch with family and friends, including those in the broad materials research community.

I start my day reading $(\sim 70)$ comic strips on my iPad. I can't go through my daily routine without two cups of coffee and life lessons from Herb and Jamaal, Mallard Fillmore, and Non Sequitur. I need to see what Beetle Bailey, Hagar the Horrible, Mary Worth, and The

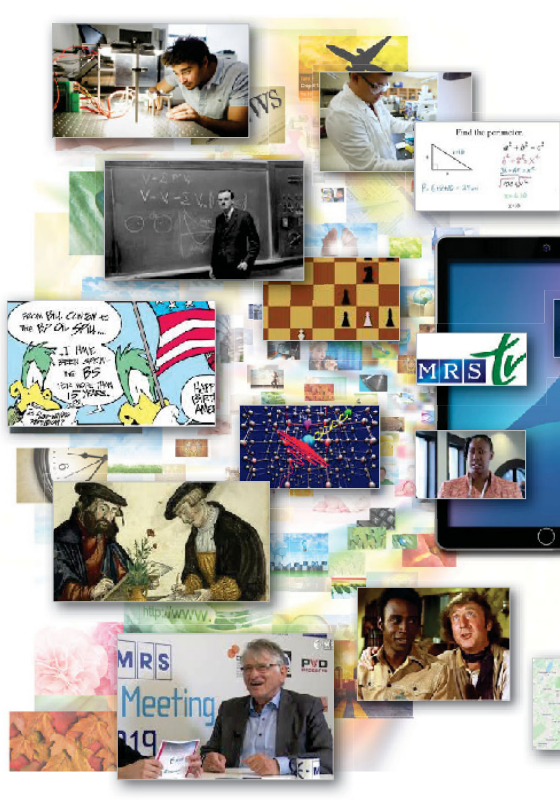
Phantom are up to. During the day, whether reading, watching TV, working on a project, or participating in a meeting, I have moments when I need additional information about topics of interest. Realtime access to this information has enabled a better understanding of events and better interactions with friends and colleagues.

The Kindle app and eBooks allow me to read for fun and to continue to learn. For instance, I am involved in a History of Science Discussion Group at the local university that picks books to read and then meets regularly to discuss them.

Each year, I attend several scientific and technical conferences, notably the Materials Research Society (MRS) Spring and Fall Meetings, and meeting apps greatly facilitate my activities. I also utilize travel apps that are lifesavers in providing needed information (delays, cancellations) in a timely fashion.

I use a cooking app that provides recipes and cooking tips. It helps me reduce waste by allowing me to efficiently plan for each meal, getting me one step closer to meeting my goal of zero wasted food. Shopping apps have aided me in buying carpet, cabinets, and other items for my new home. Maps and guidance apps aid in navigation, whether I'm traveling locally or hundreds of miles. I also routinely play games on my iPad: Bridge, Sudoku, and Chess.

YouTube allows me to view clips from movies and television shows, so that I can pinpoint my favorite scenes. I've also watched numerous clips on geometry, trigonometry, differential and integral calculus, ordinary and partial differential equations, and topology where problems related to all of these are solved. The skill levels required for the various problems range from grade school through postgraduate studies. Interested in fractional calculus? Want to know how to perform a fractional derivative or a fractional integral? What does the operator $\mathrm{d}^{\Pi} / \mathrm{dx}^{\Pi}$ mean? What does the operator w mean? Or is it nonsense? What is the value of $0^{0}$ ? What is the tetration of $\mathrm{x}$, and how do you differentiate
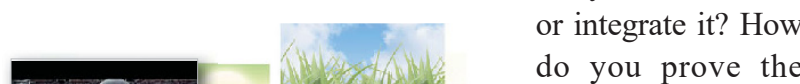
Cy $-8=0$ Pythagorean theoan $x$ rem? Is it proper to ard attribute the theorem VIV $=2 y^{2}$ c to Pythagoras? What is the significance of Ramanujan's number, 1729 ?

Did you ever wonder about how to derive Dirac's equation for the electron, or how to derive the Klein-Gordon equation? How do we know that the Schrödinger equation is nonrelativistic? What do atomic and molecular orbitals really look like? What is density functional theory, and how is it used? Countless videos are available discussing issues in the physical sciences.

Informational clips from MRS include webinars, special content (plenary speakers, Kavli lectures, Award talks), and MRS TV. Among my favorites are interviews and talks by Mildred Dresselhaus, including her Von Hippel Award talk. ${ }^{1}$ There is an interview on MRS TV with M. Stanley Whittingham (winner of the 2018 David Turnbull Lectureship Award ${ }^{2}$ ) that predates his 2019 Chemistry Nobel Prize. His talk associated with the Nobel can be found on YouTube as well. There are many other short interviews on MRS TV that are a who's who of luminaries within the materials research community

When I think back to what life was like before these technologies, those days seem surreal. Little of this would have been possible without considerable advances in materials research. I am excited by the possibility of incorporating aspects of quantum information systems or artificial intelligence into my electronic companions. Moreover, I look forward to a future with even more advances, which will come as a result of changes through materials research.

Steve Moss

1. https://www.youtube.com/watch?v=1dOMHXLcZtE.

2. https://www.youtube.com/watch?v=xWvDuoyuGp4. 